\title{
The antibody response against human and chimeric anti-TNF therapeutic antibodies primarily targets the TNF binding region
}

\author{
K A van Schie, ${ }^{1,2}$ M H Hart, ${ }^{1,2}$ E R de Groot, ${ }^{1,2}$ S Kruithof, ${ }^{1,2}$ L A Aarden, 1,2 \\ G J Wolbink, ${ }^{1,2,3}$ T Rispens $^{1,2}$
}

Handling editor Tore K Kvien

- Additional material is published online only. To view please visit the journal online (http://dx.doi.org/10.1136/ annrheumdis-2014-206237).

${ }^{1}$ Sanquin Research, Department of Immunopathology, Amsterdam, The Netherlands

${ }^{2}$ Landsteiner Laboratory, Academic Medical Centre, University of Amsterdam, Amsterdam, The Netherlands ${ }^{3}$ Jan van Breemen Research Institute | Reade, Amsterdam, The Netherlands

\section{Correspondence to} Dr Theo Rispens, Sanquin Blood Supply, Plesmanlaan 125, Amsterdam 1066 CX, The Netherlands;

T.rispens@sanquin.nl

Received 8 July 2014 Revised 15 September 2014 Accepted 5 October 2014 Published Online First 23 October 2014

\section{ABSTRACT}

Background In a subset of patients, anti tumour necrosis factor (TNF) therapeutic antibodies are immunogenic, resulting in the formation of antidrug antibodies (ADAs). Neutralising ADAs compete with TNF for its binding site and reduces the effective serum concentration, causing clinical non-response. It is however unknown to which extent ADAs are neutralising.

Objectives To study which proportion of antibodies to human(ised) anti-TNF (adalimumab, golimumab, certolizumab) as well as chimeric anti-TNF (infliximab) is neutralising.

Methods Neutralising capacity of ADAs was assessed using a TNF competition assay in ADA-positive sera of patients treated with adalimumab $(n=21)$, golimumab $(n=4)$, certolizumab $(n=9)$ or infliximab $(n=34)$ sent in to our diagnostic department.

Results In 34 sera with ADAs to adalimumab, golimumab or certolizumab, $>97 \%$ of the antibodies were neutralising. In 34 sera with ADAs to infliximab $>90 \%$ of the antibodies were neutralising. Further characterisation of the broader antibody response to infliximab revealed that non-neutralising antibodies to infliximab do not target murine domains, but may bind infliximab-unique domains not involved in TNF binding (located outside the paratope).

Conclusions Our study shows that ADAs to human (ised) as well as chimeric anti-TNF therapeutic antibodies are largely neutralising. This highly restricted ADA response suggests an immunodominant role for the paratope of anti-TNF therapeutics.

\section{INTRODUCTION}

Anti tumour necrosis factor (TNF) therapeutic antibodies are increasingly used to treat inflammatory disorders like rheumatoid arthritis and inflammatory bowel disease. Unfortunately, in some patients the therapeutics are immunogenic, resulting in the formation of antidrug antibodies (ADAs) that can lead to loss of clinical response. The percentage of patients that develop ADA to TNF blockers varies strongly between immunogenicity studies. For adalimumab, using a highly drug-tolerant antigen binding test it was found that around $53 \%$ of adalimumab-treated patients made ADA to some extent. ${ }^{1}$ In this context, two kinds of ADA can be discriminated, namely non-neutralising antibodies that bind to the drug simultaneously with TNF, and neutralising antibodies that compete with TNF for the antigen-binding site (paratope). Neutralising antibodies can therefore immediately inhibit the working mechanism of the drug. ${ }^{2} 3$

In patients treated with anti-TNF therapeutics, measuring the ADA titre as well as the drug level provides an objective view on the patient's clinical response. However, one could argue that not only the ADA titre, but also the neutralizing capacity should be monitored. For example, for patients with multiple sclerosis treated with interferon- $\beta$, neutralising antibodies rather than binding antibodies are routinely assessed, since the former better correlate with clinical impact. ${ }^{4}$ However, the neutralisation capacity is usually monitored using a bioassay, which is difficult to standardise and cumbersome to carry out.

Previous work on the antibody response to adalimumab indicates that this response is restricted to the TNF binding site (the paratope), meaning that all ADAs are neutralising. ${ }^{5}$ The antibody construct of the fully human adalimumab might explain this restricted response, since typically the majority of foreign determinants is localised in the complementarity determining regions (figure $3 \mathrm{~B}$ ). Chimeric antibodies like infliximab differ from human(ised) antibodies as the entire variable domain is of murine origin which potentially induces a broader immune response. Moreover, the variable domains of infliximab contain additional determinants that are not involved in TNF binding and are neither mouse nor human germline which might be immunogenic (figure $3 \mathrm{~A}$ ). For the anti-TNF therapeutic antibodies infliximab, certolizumab (humanised) and golimumab (human) the neutralising capacity has never been investigated.

In this study, we systematically investigated the proportion of neutralising and non-neutralising antibodies to infliximab, adalimumab, certolizumab and golimumab.

\section{METHODS}

Details about the methodology can be found in the online supplementary Materials and Methods. Briefly, patient sera from our diagnostic department were selected for ADA positivity for either adalimumab, golimumab, certolizumab or infliximab. To determine the neutralising capacity of these ADAs, a TNF competition assay was set up in which TNF is used to block the antigen binding site of radiolabelled drug. In this assay, neutralising antibodies compete with TNF for their binding to the drug, 
resulting in a decreasing signal with increasing amounts of TNF. In contrast, non-neutralising antibodies can bind the labelled drug simultaneously with TNF and the binding is not affected when TNF is added. In some experiments, polyclonal mouse IgG was added as additional blocker to examine reactivity to mouse determinants. The biological activity of anti-infliximab was analysed using the Walter and Elisabeth Hall institute (WEHI) bioassay. TNF-sensitive WEHI-164 cells were incubated in the presence of TNF, infliximab, and samples containing antiinfliximab, and viability was measured.

\section{RESULTS}

\section{ADA to all anti-TNF drugs are $>90 \%$ neutralising}

Twenty-one patients producing ADA to the fully human therapeutic antibody adalimumab were tested in a TNF competition assay (described in figure 1). Upon an increasing concentration of TNF, all patients showed an almost identical reduction in binding in a dose-dependent manner, with the highest concentrations of TNF reducing the binding to background levels (figure 2A). These results indicate that $>97.7 \%$ of ADA to adalimumab are directed to the paratope of the drug and are thus neutralising (figure 2B). This is congruent with our previous findings which suggested that antiadalimumab antibodies are highly restricted to the paratope of adalimumab. ${ }^{5}$ Similarly, for sera containing antigolimumab or anticertolizumab antibodies, respectively $>98.3 \%$ and $>97.2 \%$ inhibition of binding to golimumab and certolizumab Fab' was observed when the highest concentration of TNF was added. Thus, in 34 cases of ADA to a human(ised) anti-TNF, $>97 \%$ of the antibody response is neutralising.

For the chimeric infliximab, of which the entire variable domains are of murine origin, the results from the TNF competition assay were more varied. The ADA of one group of patients showed a decrease in signal with an increase in TNF leading to essentially complete suppression of ADA binding to the drug on the end point of the TNF titration. In contrast, a second group of patients showed incomplete inhibition of ADA binding to the drug when the highest concentration of TNF was added. The sera of this latter group thus contain ADA that are able to bind the drug simultaneously with TNF and are therefore non-neutralising. Nevertheless, all patient sera tested

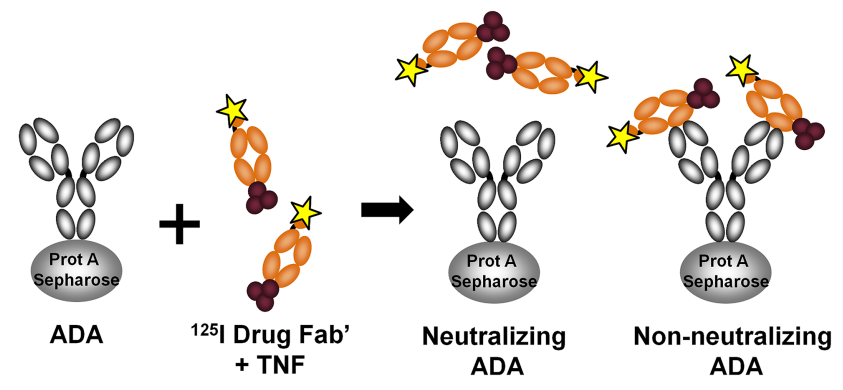

Figure 1 Schematic representation of the TNF competition assay. To determine the neutralising capacity of antidrug antibodies (ADAs), a TNF competition assay was set up in which tumour necrosis factor (TNF) is used to block the paratope (or antigen binding site) of the labelled drug. Serum IgG including ADA is bound by protein A sepharose, and radiolabelled drug Fab' will subsequently bind to ADA. However, upon addition of TNF, the paratope of the radiolabelled drug Fab' is blocked by TNF. Consequently, ADAs that bind (close to) the paratope of the drug cannot bind anymore and are classified as neutralising ADAs. ADAs that can bind simultaneously with TNF are classified as non-neutralising. contained $>90 \%$ neutralising ADA to infliximab. In line with these observations, ADA titres to infliximab determined in the WEHI bioassay, which only measures the effect of neutralising $\mathrm{ADA}$, are in good correlation with those determined in the antigen binding test (ABT) which measures all ADA (figure 2C). No relationship was found between ADA levels and the percentage of neutralising antibodies (see online supplementary figure S2). In conclusion, for all TNF blockers, the vast majority of ADAs neutralise the drug, in case of human(ised) antibodies and for the chimeric infliximab.

\section{Incomplete inhibition of ADA to infliximab is not caused by ADA-drug complexes}

Although no free infliximab was detected in any of the sera, the possibility exists that complexes of infliximab and antiinfliximab are present. Hypothetically, if these complexes dissociate, a false-positive signal may result, since infliximab can bind protein A sepharose and TNF can subsequently form a bridge between bound infliximab and radiolabelled infliximab Fab. To exclude the possibility that the non-inhibitable signals were the result of drug-ADA complexes, monomeric $\operatorname{IgG}$ was separated from antibody complexes using fast protein liquid chromatography (FPLC) (see online supplementary figure S1A). However, no differences in neutralisation were found between the monomeric fractions compared with patient serum (see online supplementary figure $\mathrm{S} 1 \mathrm{~B}$ ). This indicates that the incomplete inhibition of ADA to infliximab by TNF observed in some patients is not caused by ADA-drug complexes, but in fact are non-neutralising anti-infliximab antibodies.

\section{Non-neutralising ADA to infliximab do not target mouse determinants}

To investigate whether the non-neutralising anti-infliximab antibodies target predominantly infliximab unique determinants outside the paratope or mouse germline determinants foreign to humans (figure $3 \mathrm{~A}$ ), we modified the TNF competition assay by the addition of polyclonal mouse IgG. In theory, nonneutralising ADAs that target mouse germline epitopes are blocked by mouse IgG, thereby inhibiting binding of radiolabelled infliximab Fab seen as a decrease in signal. However, in serum samples containing non-neutralising antibodies, we observed no additional inhibition when mouse $\operatorname{IgG}$ was added (figure 3C,D); this suggests that non-neutralising anti-infliximab antibodies are not directed to mouse germline determinants of infliximab, but target determinants outside the paratope that are unique to infliximab.

\section{DISCUSSION}

We demonstrated that ADAs to the human(ised) antibodies adalimumab, golimumab and certolizumab were highly confined to the paratope (>97\%). Infliximab elicited a slightly broader immune response, although in all patients at least $90 \%$ of all ADAs were neutralising. In addition, a strong correlation was found between the anti-infliximab titre determined by ABT, in which all ADAs are detected, and the WEHI bioassay, which only measures neutralising ADAs. These results indicate that conventionally measured titres of binding antibodies closely resemble titres of neutralising ADAs. Therefore, measurement of neutralising antibodies to these TNF blockers, for example, by means of a bioassay, is not useful.

Sera containing low ADA levels (below $100 \mathrm{AU} / \mathrm{mL}$ ) were not investigated, because the low levels preclude accurate quantification of the fraction of neutralising antibodies. The sera that 
A Adalimumab Golimumab Certolizumab Infliximab

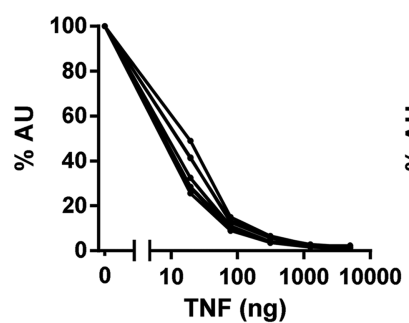

B

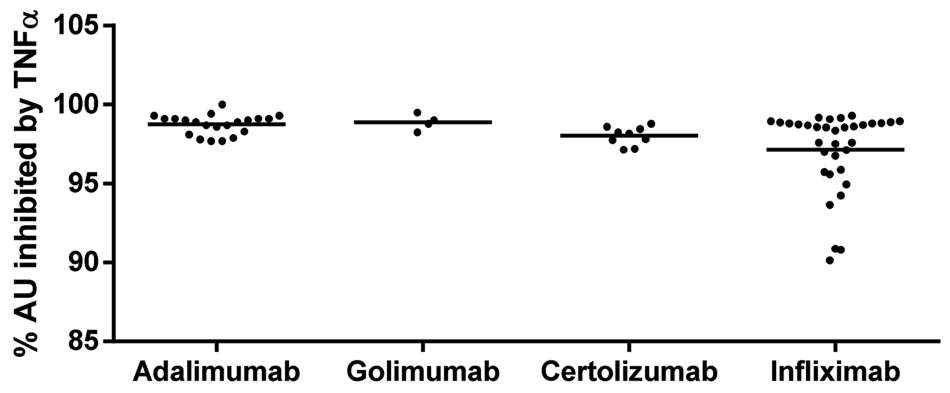

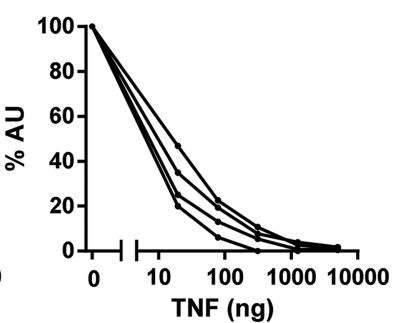

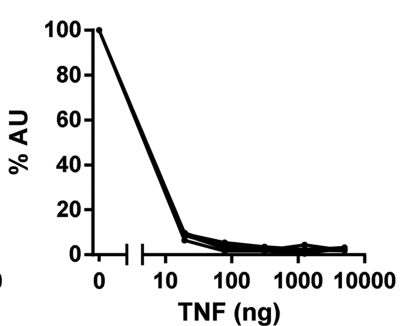

C

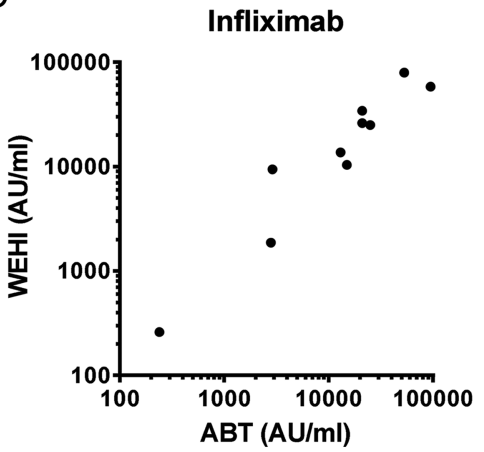

Figure 2 More than $90 \%$ of antidrug antibodies (ADAs) to all anti tumor necrosis factor (TNF) therapeutic antibodies target the paratope of the drug. (A) Radiolabelled drug preincubated with increasing amounts of TNF gives a decrease in binding of ADA to the drug. ADAs to all four anti-TNF therapeutic antibodies were $>90 \%$ neutralising. Each line represents a single patient. In case of adalimumab, certolizumab and infliximab, a selection of representative patients is shown. (B) Percentage of AU inhibited by $5000 \mathrm{ng}$ of TNF. Each dot represents a single patient. Median and range of adalimumab 98.9 (97.7-100), $\mathrm{n}=21$; golimumab 98.9 (98.3-99.5), $\mathrm{n}=4$; certolizumab 98.17 (97.2-98.8), $\mathrm{n}=9$; infliximab 98.6 (90.2-99.3), $n=34$. (C) Anti-infliximab titres were measured using the ABT and the WEHI bioassay. The ABT measures all ADAs whereas the WEHI bioassay only measures neutralising ADAs. The strong correlation indicates that all ADAs are neutralising. Spearman's $\rho$ was $r=0.9362(p=0.0002, n=10)$.

were tested comprise a broad range of ADA levels that do not correlate with the percentages of neutralising antibodies (see online supplementary figure S2), However, a possibly broader response in patients with low ADA levels cannot be excluded, although absolute titres of non-neutralising ADAs will necessarily be low in such cases.

This study focused on IgG antibodies, since these were previously found to represent the predominant class of antibodies formed to infliximab or adalimumab. ${ }^{6}{ }^{7}$ Moreover, IgM antibodies typically have low affinity and are poor in neutralising the drug, while IgG antibodies have high affinity and are therefore more potent drug inhibitors.
It was already known that anti-infliximab antibodies may neutralise the drug. ${ }^{6}$ We however observed that the majority $(>90 \%)$ of antibodies to infliximab are neutralising, clearly demonstrating that the paratope of infliximab is immunodominant. Given the high number of non-human determinants in the variable domains of infliximab (figure 3A) this is a remarkable observation. Interestingly, paratope immunodominance was already suggested in an earlier study of the antibody response to the therapeutic mouse monoclonal antibody OKT3, where it was estimated that $50-60 \%$ of all ADAs were targeting the paratope. ${ }^{8}$ Moreover, in a more recent study, binding of minipig antibodies to adalimumab could not be inhibited by human

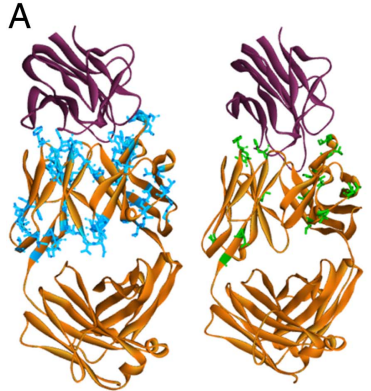

Infliximab

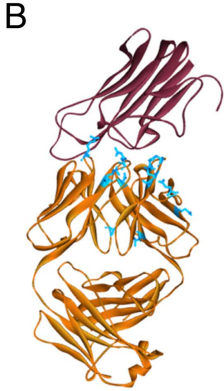

Adalimumab
C

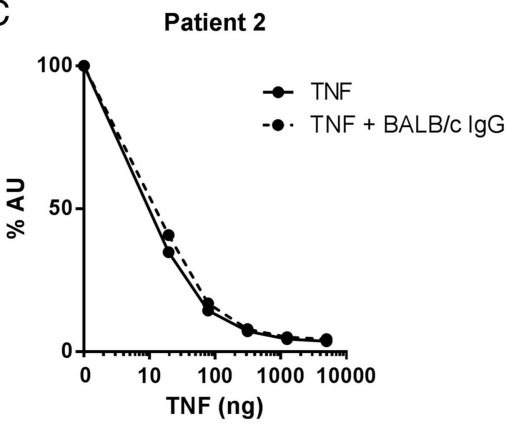

D

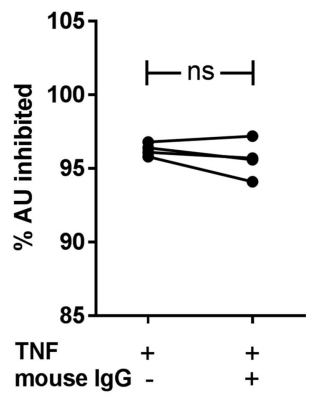

Figure 3 Non-neutralising antidrug antibodies to infliximab do not target mouse determinants. Crystal structure of infliximab (A) or adalimumab. (B) Fab (orange) in complex with tumor necrosis factor (TNF) (purple). Residues that differ from human germ line are highlighted in blue. Residues that are unique to infliximab (ie, differ from mouse germ line) are highlighted in green. Figures rendered from PDB coordinate files 4G3y (infliximab) $^{10}$ and 3WD5 (adalimumab), ${ }^{11}$ aligned using DomainGapAlign (available from the ImMunoGeneTics website). (C and D) Sera of four patients that showed incomplete inhibition by TNF were tested for antibodies to mouse determinants of infliximab. Polyclonal mouse lgG was added to the TNF competition assay to block antimouse antibodies. No inhibition could be observed. ns=not significant using paired $t$ test. 
polyclonal IgG $\mathrm{F}(\mathrm{ab})_{2}$, suggesting anti-idiotypic antibodies. ${ }^{9}$ These results highlight that reducing immunogenicity in anti-TNF therapeutic antibodies will remain a challenging endeavour.

Surprisingly, the non-neutralising fraction of anti-infliximab antibodies that was found in approximately a third of the patients did not target mouse germline determinants, since addition of polyclonal mouse IgG did not reduce binding of ADA to the drug. Aligning the amino acid sequence of the infliximab variable domains ${ }^{10}$ with the mouse germline sequence revealed several accessible non-mouse germline (infliximab unique) amino acids that were not part of the paratope but could be targeted by the immune response (figure $3 \mathrm{~A}$ ). It is therefore likely that non-neutralising anti-infliximab antibodies target determinants that are unique to infliximab, but not involved in TNF binding.

In summary, only for the chimeric therapeutic infliximab, but not for the human(ised) adalimumab, golimumab and certolizumab, a small amount of non-neutralising antibodies could be observed, which implies that the closer the drug is to the human germline, the more restricted the antibody response to it is. Nevertheless, in all cases $>90 \%$ of all ADAs were neutralising, which shows that the TNF binding region of anti-TNF therapeutic antibodies is immunodominant even in case of the chimeric antibody infliximab.

Acknowledgements The authors thank Henk de Vrieze for his technical support.

Contributors Study concept and design: KAvS, LAA, GJW, TR. Acquisition of data: KAvS, MHH, ERdG, SK. Analysis and interpretation of data: KAvS, MHH, ERdG, SK, LAA, GJW, TR. Obtained funding: GJW. Study supervision: LAA, GJW, TR.

Funding Funding of this study was provided by an unrestricted grant from Pfizer. Pfizer had no involvement in the study design; in the collection, analysis, and interpretation of data; in the writing of the manuscript; or in the decision to submit the manuscript for publication.
Competing interests GJW has received a research grant from Pfizer and honoraria for lectures from Abbvie, Pfizer and UCB. LAA has received honoraria for lectures from Abbott, Roche and Pfizer. TR has received honoraria for lecture from Pfizer and Abbvie.

Provenance and peer review Not commissioned; externally peer reviewed.

\section{REFERENCES}

1 Van Schouwenburg PA, Krieckaert CL, Rispens $\mathrm{T}$, et al. Long-term measurement of anti-adalimumab using $\mathrm{pH}$-shift-anti-idiotype antigen binding test shows predictive value and transient antibody formation. Ann Rheum Dis 2013;72:1680-6.

2 Bartelds GM, Krieckaert CLM, Nurmohamed MT, et al. Development of antidrug antibodies against adalimumab and association with disease activity and treatment failure during long-term follow-up. J Am Med Assoc 2011;305:1460-8.

3 Bendtzen $\mathrm{K}$, Geborek P, Svenson M, et al. Individualized monitoring of drug bioavailability and immunogenicity in rheumatoid arthritis patients treated with the tumor necrosis factor alpha inhibitor infliximab. Arthritis Rheum 2006:54:3782-9.

4 Paolicelli D, D'Onghia M, Pellegrini F, et al. The impact of neutralizing antibodies on the risk of disease worsening in interferon $\beta$-treated relapsing multiple sclerosis: a 5 year post-marketing study. J Neurol 2013;260:1562-8.

5 Van Schouwenburg PA, van de Stadt LA, de Jong RN, et al. Adalimumab elicits a restricted anti-idiotypic antibody response in autoimmune patients resulting in functional neutralisation. Ann Rheum Dis 2013;72:104-9.

6 Kosmac M, Avcin T, Toplak N, et al. Exploring the binding sites of anti-infliximab antibodies in pediatric patients with rheumatic diseases treated with infliximab. Pediatr Res 2011;69:243-8.

7 Svenson M, Geborek P, Saxne T, et al. Monitoring patients treated with anti-TNF-alpha biopharmaceuticals: assessing serum infliximab and anti-infliximab antibodies. Rheumatology (Oxford) 2007;46:1828-34.

8 Chatenoud L, Baudrihaye MF, Chkoff N, et al. Restriction of the human in vivo immune response against the mouse monoclonal antibody OKT3. J Immunol 1986;137:830-8. http://www.ncbi.nlm.nih.gov/pubmed/3487589

9 Van Mierlo GJD, Cnubben NHP, Wouters D, et al. The minipig as an alternative non-rodent model for immunogenicity testing using the TNF $\alpha$ blockers adalimumab and infliximab. J Immunotoxicol 2014;11:62-71.

10 Liang S, Dai J, Hou S, et al. Structural basis for treating tumor necrosis factor $\alpha$ (TNF $\alpha$ )-associated diseases with the therapeutic antibody infliximab. I Biol Chem 2013;288:13799-807.

11 Hu S, Liang S, Guo H, et al. Comparison of the inhibition mechanisms of adalimumab and infliximab in treating tumor necrosis factor $\alpha$-associated diseases from a molecular view. J Biol Chem 2013;288:27059-67. 